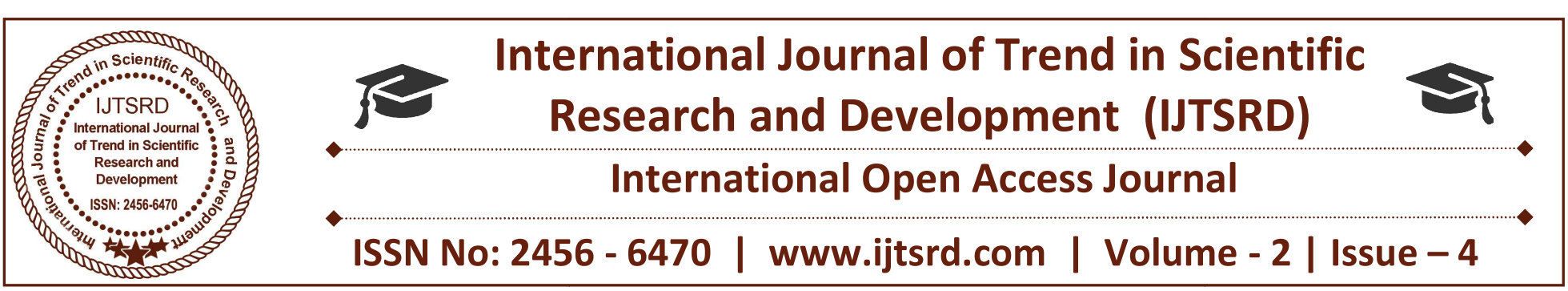

\title{
Spy Detection for Android Devices
}

\author{
Deepthi S Kumar ${ }^{1}$, Naren M $S^{2}$, Nishita K Murthy ${ }^{3}$, Soumya K $\mathbf{N}^{4}$ \\ ${ }^{1,2,3}$ B.Tech Final Year, ${ }^{4}$ Assistant Professor, \\ Department of Information Science \& Engineering \\ Jain University - School of Engineering \& Technology Karnataka, India
}

\section{ABSTRACT}

This paper proposes a procedure to enhance anti- theft to prevent robbery for android-based smartphones by utilizing SMS as a medium. As the usage of phones, tablets, note pads are designed with the help of android OS is drastically rising and expanding, numerous situations related with anti- theft have additionally been evolved, however the greater part of the software products are not readily available and it is hard to recognize the robber by utilizing these software products like GPS Tracking. We have set forward another plan, which improves the current situation, in view of new advancements like messages being sent and received. The situation proposed in this work is absolutely reliant on the hardware of the cell phone like camera (front) and GPS tracker. When this application is installed on the phone, it will work in the background without displaying the application to the thief, stores the current SIM number in a server and application and continues checking continuously for the change in the SIM. Once the SIM card allotted on the device changes, it will capture 3 pictures and the GPS co- ordinates (latitude and longitude) are obtained. The details are acquired without taking the permission from the user. The pictures and GPS coordinates are stored in the server and an SMS is sent to an alternate number which was given during login. The major benefit of this product is that it is very simple to configure, it continues running as a background application without the thief's notice and without interfering with the user. It assists the owner to identify the criminal on his own.
Keywords: Android, Smartphones, Subscriber Identity Module (SIM), Global Positioning System (GPS), Web Application

\section{INTRODUCTION}

The most recent cell phones, for example, Androidbased cell phones, called smartphones, are changing the way we experience our lives and has turned into an essential piece of our lives. Cell phones change the methods for communication not like landline telephones, it is advantageous for virtual speaking with anyone basically through video-conferencing, email, and so forth., and it likewise gives a provision to store contact numbers and email in the phone memory which decreases the idea of File- System to store individual contacts.

These days, cell phones are acting like a personal computer, it can be utilized to store data, documents and so forth., and can be distributed to anybody through the Internet. These most recent cell phones are exceptionally useful for working together in a business. Organization related data and reports can be seen from anyplace and can be shared with anybody.

\section{LITERATURE SURVEY}

\section{Anti-Theft Mobile Phone Security System with The Help of BIOS}

This paper proposes a technique where basic input and output system is used in order to develop an antitheft security feature for phones. This cell phone security framework enables us to decide the location of cell phone. The proposed security framework 
depends on hardware execution procedure in which the phone is composed such that a phone can be followed out regardless of whether battery and Subscriber Identity Module (SIM) are removed from the device. Besides, we likewise think about the utilization of BIOS and its significance in our day by day life. The proposed methodology will help the originators in enhancing the gadget security.

As indicated by the current trend in the market and the various observations, the stolen cell phones are kept in the OFF state or the thief can plug its battery (or SIM) out. In such circumstances, one cannot find the location of the gadget? Numerous creators introduced thoughts to localize the gadget and every one of these thoughts are not effective when there is no power supply. A gadget can be found just around then when it is in ON state. A few of the prominent smartphone tracking frameworks created are as per the following:

- Android Device Manager (ADM)

- Apple’s Security System (ASS)

- Find my Phone (FMP)

- Tracking theft mobile application (TTM) Here, a Tracker SIM that is embedded in the device as an implicit BIOS cell for longer time. The Tracker SIM trades GPS location co-ordinates (latitude and longitude) with base station through an antenna to receive signals which is worked through the BIOS cell in OFF state. The proposed arrangement will help the creators in enhancing the security of the device.

\section{Mobile Location Estimation and Tracking for GSM Systems}

This paper proposes a phone location estimation and strategy for tracking for remote correspondence systems. The area estimation depends on the distinctions of down-link signal disturbances (attenuation), which are utilized to decide the number of cycles created by the various possible device location. At that point, the genuine area is given by the crossing point (intersection) of the circles. The considerable advantage of this strategy is to obtain the accurate location and path, and to reduce the effect of shadows. Also, by using the tracking technique through piece-wise linear optimization utilizing a straightforward genetic calculation is connected to enhance the areas estimation. As the outcomes are appeared, the estimation faults (are significantly littler than the mistakes from cell-ID strategy in a genuine GSM framework).

\section{Hybrid Location Estimation and Tracking System for Mobile Devices}

Smartphone location estimation has pulled in loads of user-attention lately. The algorithms for location used on the cell phones can usually be sorted into the satellite based also, the network-based frameworks.

The two kinds of framework have their favourable circumstances and restrictions under various situations (i.e. urban, rural, or country region). To provide adjustment to different situations for location estimation, a hybrid schema to detect location, which is a combination of both the network-based and the satellite-based signals, is proposed in this paper.

The proposed method uses the two-step Least Square technique for assessing the three- dimensional position (i.e. the longitude, latitude, and altitude) of the cell phones. The Kalman filtering method is used to remove the noises during measurement and to track the directions of the cell phones.

An algorithm to perform fusion is utilized to acquire the final location estimation from both the satellitebased and the network-based frameworks. Numerical outcomes exhibit that the proposed hybrid location technique gives exact location estimation by adjusting under various conditions.

\section{AALTm: An Android Application to Locate and Track Mobile Phones}

This paper depicts a tracking application called AALTm (An Android Application to Locate and Track Mobile phones) which is installed with a ton of highlights, for example, area following, SIM card recognition, call observing, profile changing, sending the pictures to the predefined email address and erasing the sent and received messages. These highlights are very unique in relation to the current tracker applications which would be useful in following the lost smartphone.

The AALTm can turn on the GPS when a nonapproved SIM card is figured out in the mobile by contrasting the Integrated Circuit Chip Card Identification (ICC ID). The ICC ID number is special (unique) for each SIM card. 


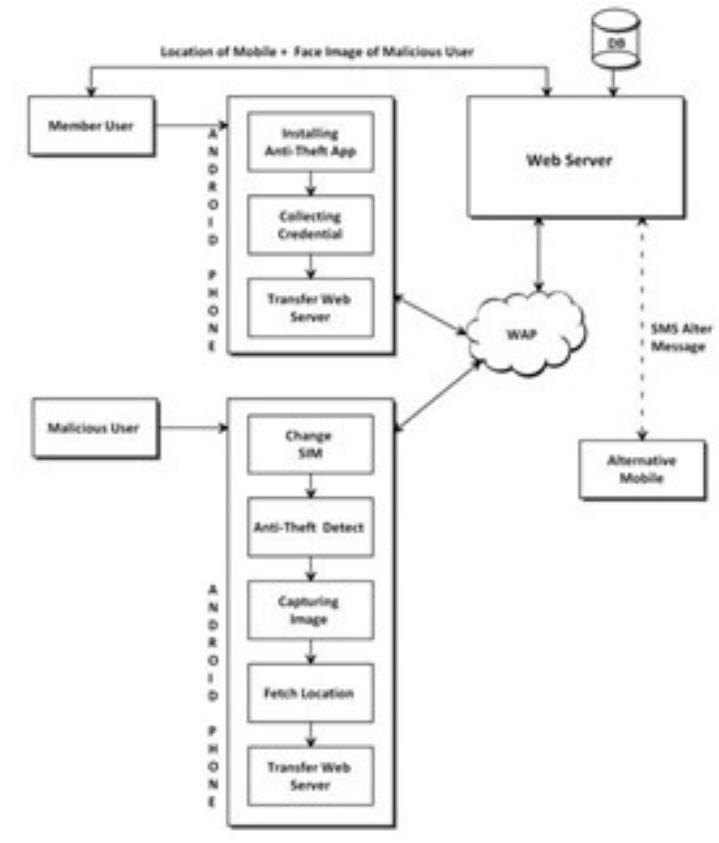

Figure 3.1: System Architecture

\section{SYSTEM ARCHITECTURE}

The system architecture includes:

The role of the user includes:

- On the Android phone, the Anti-theft app should be installed initially.

- The credentials such as phone number and alternate phone number should be entered by the user

- These details are stored in the server

The activities performed by the malicious user (thief) are:

- The sim on the stolen phone will be changed by the thief

- As soon as the sim in changed, the Anti- theft application starts running on the phone

- The application captures 3 images and fetches the location of the phone using the GPS

- These details are stored in the database and can be used to track down the thief

- The web server sends the location and the captured images to the user

A message is sent to the alternate number saying the phone has been stolen
In this system, images of the thief and location of the stolen device are sent to the server for further monitoring. As soon as change in SIM is detected, a message is sent to the registered alternate phone number. The spy images are clicked and the GPS location is detected and sent to the server. These details are available for the respective user only. The location and the pictures can be used to track down the thief.

The various objectives that we tried achieving in this paper are:

- Images of the thief and location of the stolen device are sent to the server for further monitoring.

- As soon as change in SIM is detected, a message is sent to the registered alternate phone number.

- The spy images are clicked and the GPS location is detected and sent to the server.

- These details are available for the respective user only.

The location and the pictures can be used to track down the thief.

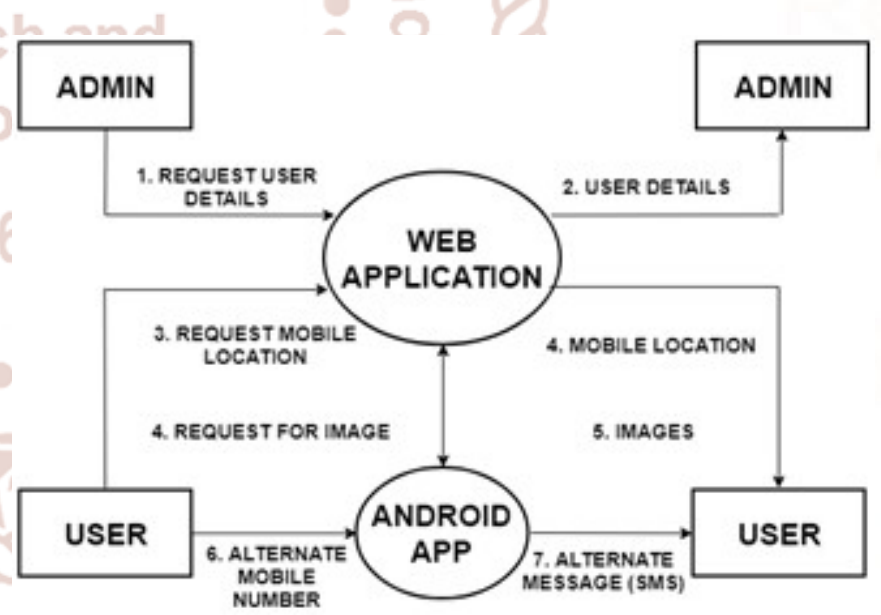

Figure 4.1: Block Diagram of Spy Detection for Android Devices

The process includes:

- The admin is requested to enter his details to store it in the database.

- The user details are stored as well. He is asked to enter details like name, phone number, alternate phone number, user password and email ID. 
- This can be viewed by the admin

- In case of any theft, the application is designed in such a way where the images are captured and the location is detected of the thief who stole the smartphone.

- The detected location and the images are sent to the user from the phone which can be viewed on the web application

- In the Android part, the owner of the phone needs to install the application on the phone and enter his sim details along with an alternate contact number

- The alternate contact number receives a message regarding the stolen phone. This can help in tracking down the thief at the right location.

\section{CONCLUSION AND FUTURE SCOPE}

This project presents a novel anti-theft spy detect application for android based devices. The application deploys an enterprise security solution that meets user's immediate and long-term requirements by providing the images of the thief, which makes it easy for the user to identify the thief and make him/her surrender and get arrested. Also, we are enhancing this application by providing the information about the location of the android smartphone and sending an alert text message to alternate number. With the advent of time, technology is evolving. Our goal is to improve and update the application timely. Currently this application is available for android based mobile phones.

Future work involves development of the application for iOS, Symbian, Windows Mobile OS etc.

Future scope of this project is to make this as a system application and integrate it with the operating system of a smartphone and make it more seamless and secured.

\section{REFERENCES}

1. Abdul Waheed, Muhammad Riaz, and M. Yaqoob Wani, "Anti-Theft Mobile Phone Security System with The Help of BIOS", 2017.

2. Ding-Bhg Lin, Rong-Terng Juang, and Hsin-Piao Lin, "Mobile Location Estimation and Tracking for GSM Systems", 2014.
3. Chao-Lin Chen and Kai-Ten Feng, "Hybrid Location Estimation and Tracking System for Mobile Devices", 2015.

4. Merlin Monisha. A, "Anti-Theft Application to Track and Locate Lost or Stolen Android Based Mobile Devices", 2017.

5. Sonia C.V, Dr. A. R. Aswatha, "AALTm: An Android Application to Locate and Track Mobile Phones", 2014.

6. Murat Ali Bayir, Murat Demirbas, and Ahmet Cosar, "Track ME! A Web Based Location Tracking and Analysis System for Smart Phone Users", 2016.

7. Shan Chang, Ting Lu, Hui Song, "SmartDog: Real-time Detection of Smartphone Theft", 2016.

8. Martin Hellebrandt and Rudolf Mathar, "Location Tracking of Mobiles in Cellular Radio Networks", 2013.

9. Prof. Kishore N. Shedge, Dnyaneshwar C. Dhatrak, Kanchan S. Ugale, Asmita P. Walimbe, Anil S. Khade, "Mobile theft tracking application”, 2017.

10. Meng Jin, Yuan He, Dingyi Fang, Xiaojiang Chen, Xin Meng, and Tianzheng Xing, "iGuard: A Real-Time Anti-Theft System for Smartphones", 2018.

11. Bingfei REN, Yi SUN, Yunong LIN, "Anti- theft and tracking loop model based on PC and smart phone", 2013.

12. Luís C. M. Varandas, Binod Vaidya, Joel J.

13. P. C. Rodrigues, "mTracker: A Mobile Tracking Application for Pervasive Environment”, 2010.

14. R. Danu, "Tracking theft mobile application", 2016.

15. Azeem Ush Shan Khan, Mohammad Naved Qureshi, Mohammed Abdul Qadeer, "Anti- theft application for android based devices", 2014. 